\section{Day care surgery: Do we and our patients need it?}

Lorne R. Hatch MD
Early this century hospital oriented "in and out" surgical care evolved and was done in hospital integrated, hospital adjacent or satellite facilities. These concepts were controversial but utilised increasingly as costs of inpatient care continued to rise.

The first freestanding facility was established by Ford and Reed in Phoenix, Arizona. It was two years in operation before the costs were covered and this was only after assistance from insurance companies who rewrote contracts to enable payment for procedures in such a facility. Patients and insurers benefited from reduced costs and the facility flourished.

Cloud notes that " 35 per cent of 22 million surgical procedures in the U.S.A. could be done as outpatient procedures" and " 60 per cent of paediatric surgeons" work could be done as outpatient procedures." Presently about six per cent of surgery is done as an outpatient basis in the United States. In the University of Alberta Hospital, Edmonton, five to ten per cent of surgery has been done as outpatient procedures over the past three years.

Day care surgery offers certain advantages to patients, staff and the community (Table I). However, there are disadvantages too. The time for establishing rapport with patients is decreased. Even the existence of an anaesthesia clinic for consultation purposes does not necessarily overcome this and there may be an undesirable fragmentation of patient contact with medical and nursing personnel. There is also a reduced time for the observation and evaluation of postoperative complications that may occur. In the case of day care surgery the patient and the procedure are selected with the prediction that a life threatening complication will not occur once the patient has been discharged from the day care surgical unit.

The claim of reduced cost for medical care has been a major fact popularising this form of care. These have to be computed with great care if an
TABLE I Advantages of day care surgery

- Reduced cost of medical care.

- Avoidance of the depersonalising experience of hospitalisation.

- Reduced exposure to infections.

- Less time away from work.

- Less opportunity for unnecessary medical investigations.

- Rapid reduction of existing surgical lists.

accurate prediction of financial saving is to be made. The main advantage to taxpayer and insurance companies is that costs for surgical service are decreased, but inpatient admissions do not necessarily diminish as day care surgery use increases. Spinoza has written "Nature abhors a vacuum." If beds in a hospital exist, physicians will lay claim to them and do their best to keep them filled. In any case, empty beds do not cost zero dollars to maintain but 80 per cent of the cost of a bed being utilised for patient care. The total utilisation of the hospital with surgical day care units increases and thus total hospital cost may increase, although the community may be getting more medical care for its tax dollar. Accordingly, there is likely to be only a long-term regional or community financial saving during the time that the population increases but fewer inpatient beds are made available.

In conclusion, we can expect an increasing proportion of surgical patients to be cared for in a day care surgery facility and future pharmacolog. ical developments are likely to play an important role in this evolution of anaesthetic practice.

Lorne R. Hatch, MD, Honorary Clinical Instructor, University of Alberta, Department of Anaesthesia, University of Alberta Hospital, Edmonton, Alberta. 


\section{References}

1 Woo SW (Ed.). Ambulatory anesthesia care. International Anesthesiology Clinics 20: No. 1 (1982).

2 Evans RG, Robinson GC. Surgical day care: measurements of economic payoff. Can Med Ass J 1980; 123: 873.
3 Barton, ND. Outpatient surgery and anesthesia. Primary Care 1977; 4: 20.

4 Loder $R E$. The anaesthetist and the day care surgery unit. Anaesthesia 1982; 37: 1037.

5 Spinoza B. Ethics 1677. Part I, Proportion 15.

\section{Selection of patients for day care surgery}

Outpatient surgical care provides a measure of control over the ever-increasing demand for hospital beds and some relief from the soaring costs of hospitalization to the patient and society. However, to be successful there must be effective co-operation between surgeon, anaesthetist, and patient. The operating room committee representing anaesthetists and surgeons must agree on criteria for patient selection and a method for arranging consultation about special cases. Only in this way can annoyirig confrontation between surgeon and anaesthetist be avoided and the greatest comfort and safety of the patient be likely to be achieved.

\section{Patient selection}

Currently most anaesthetists will accept patients in Class I and II of the American Society of Anesthesiologists Physical Status rating. Many services will also accept Class III patients. Patients in this group must be medically stable, unlikely to be affected adversely by the proposed surgery or anaesthesia, and for whom hospital admission may be detrimental.

Outpatients must have access to the same standard of preoperative assessment provided for inpatients. A medical history must be taken and physical examination performed in a time frame that permits appropriate laboratory investigations and is not hopelessly out of date by the time the selected patient is admitted for surgery. This period varics depending on practices but should not exceed a month. Should a patient's medical status change in the interval it is essential that the proposed procedure be reconsidered.
Other very important factors in the selection of patients for day care surgery are the ability of the patient to understand and comply with instructions, the capability of the person accompanying the patient, and the distance the patient has to travel home. These factors are related in part to the surgical procedure which will be discussed later. The patient must be attended by an adult person who understands and will carry out appropriate instructions such as those described in Table I. In an urban setting the physician has more latitude when selecting patients suitable for outpatient surgery because it can be assumed that appropriate medical services upon reaching home are readily available. If the patient has a long journey home after discharge, consideration must be given to the likelihood of haemorrhage, the severity of pain, airway problems, and the quality of care while travelling. Generally, it is advisable to advise out-of-town patients and their associate to spend the first postoperative night within a reasonable distance of the surgical facility so that an urgent consultation can be rapidly obtained. The surgeon and anaesthetist should emphasize the wisdom of such an arrangement prior to the patient's admission to the surgical unit.

\section{Selection of operative procedure}

A wide variety of surgery can be done on an

R.A. McTaggart, MD, Honorary Assistant Clinical Ptofessor, University of Alberta. Department of Anaesthesia, Royal Alexandra Hospital, Edmonton, Alberta. 